\title{
Effects of frequency and feeding time on growth, food utilization, somatic indexes, and survival of juvenile white snook Centropomus viridis
}

\section{Efecto de la frecuencia y la hora de alimentación sobre el crecimiento, la eficiencia alimenticia, los índices somáticos y la supervivencia en juveniles de robalo blanco Centropomus viridis}

\author{
María Isabel Abdo-de la Parra ${ }^{1 *}$, Luz Estela Rodríguez-Ibarra ${ }^{1}$, Leonardo Ibarra-Castro ${ }^{1,2}$, \\ Juan Manuel Martínez-Brown ${ }^{1,3}$, Gabriela Velasco-Blanco ${ }^{1}$ \\ ${ }^{1}$ Centro de Investigación en Alimentación y Desarrollo, Unidad Mazatlán, Av. Sábalo Cerritos, s/n, Estero del \\ Yugo, 82112, Mazatlán, Sinaloa, Mexico. \\ 2 Whitney Laboratory for Marine Bioscience, University of Florida, 9505 Oceanshore Blvd, St. Augustine, \\ Florida 32080, USA. \\ ${ }^{3}$ Consejo Nacional de Ciencia y Tecnología, Av. Insurgentes Sur 1582, Col. Crédito Constructor 03940, \\ Mexico City, Mexico. \\ * Corresponding author. E-mail: abdo@ciad.mx
}

\begin{abstract}
The Pacific white snook, Centropomus viridis, is considered a species with high farming potential in Mexico due to its high economic value and overall demand in the national market. Growth in farmed fish is largely determined by the dietary regimen, which includes feeding frequency, rate, time, and cycle. The aim of the present study was to determine the feeding frequency and appropriate feeding time for farmed C. viridis juveniles in order to optimize growth and survival. Juveniles weighing $0.36 \pm 0.01 \mathrm{~g}$ were used to evaluate 1 and up to 5 ad-libitum feeding frequencies per day, with 3 to 24-h intervals, for 6 weeks. Gained weight (GW), growth rate (GR), specific growth rate (SGR), food conversion ratio (FCR), feeding efficiency rate (FER), coefficient of variation (CV), hepatosomatic index (HI), peritoneal fat index (PFI), and survival (S) were determined for juveniles. Regardless of the feeding hours, no significant differences were found in the GR and SGR values between juveniles fed 3 times a day and those fed 5 times a day. FCR was significantly higher and FER significantly lower in treatments with juveniles fed only once a day compared with the rest of the treatments. No significant differences were found in CV, HI, and $\mathrm{S}$ between treatments. PFI was significantly different only between juveniles fed once and 5 times a day. Considering the results obtained in this study, we suggest feeding C. viridis juveniles 3 times a day at 6-h intervals between doses. These results will contribute to the development of biotechnology for farming this species.
\end{abstract}

Key words: feeding regimen, growth, juvenile white snooks, Centropomus viridis.

RESUMEN. El robalo blanco del pacífico, Centropomus viridis, se considera como una especie con alto potencial para su cultivo en México por su alto valor económico y gran demanda en el mercado nacional. El crecimiento de los peces en cultivo depende, en gran medida, del régimen alimenticio, el cual incluye, frecuencia, tasa, tiempo y ritmo de alimentación. El presente trabajo tuvo como objetivo determinar la frecuencia y la hora apropiada de alimentación de juveniles de $C$. viridis en cultivo para optimizar el crecimiento y la supervivencia. Se utilizaron juveniles de $0.36 \pm 0.01 \mathrm{~g}$ para evaluar de 1 a 5 frecuencias de alimentación ad libitum por día, con intervalos de 3 a 24 h, durante 6 semanas. Se determinó el peso ganado (PG), la tasa de crecimiento (TC), la tasa específica de crecimiento (TEC), la tasa de conversión alimenticia (TCA), la tasa de eficiencia alimenticia (TEA), el coeficiente de variación (CV), el índice hepatosomático (IH), el índice de grasa peritoneal (IGP) y la supervivencia (S) de los juveniles. La TC y la TEC de los juveniles alimentados 3 veces al día, independientemente de las horas de alimentación, no presentaron diferencias significativas con respecto a los resultados obtenidos para los juveniles alimentados 5 veces al día. La TCA fue significativamente mayor y la TEA significativamente menor en los tratamientos con juveniles alimentados una sola vez al día en comparación con el resto de los tratamientos. No se encontraron diferencias significativas en CV, IH y S entre los tratamientos. El IGP solo presentó diferencias significativas entre los juveniles alimentados 1 y 5 veces al día. Con base en los resultados obtenidos en el presente estudio, se sugiere que los juveniles de $C$. viridis se alimenten 3 veces al día, con un intervalo de 6 h entre cada toma. Estos resultados ayudarán en el desarrollo de la biotecnología de cultivo de esta especie.

Palabras clave: régimen alimenticio, crecimiento, juveniles de robalo blanco, Centropomus viridis. 


\section{INTRODUCTION}

The Pacific white snook (Centropomus viridis) has a distribution that ranges from Baja California Sur and the Gulf of California to southern Ecuador and the Galapagos Islands (Fischer et al. 1995). Species in the Centropomidae family inhabit shallow coastal waters, estuaries, rivers, and brackish lagoons and migrate to fresh waters. Juveniles and pre-adults are commonly found in mangrove areas, where they show great tolerance to salinity fluctuations given their osmoregulatory capacity (Álvarez-Lajonchère et al. 2013). Snooks are considered species with high farming potential in Mexico because of their high economic value and overall demand in the national market given the flavor, color, and texture of their meat; their high growth rates; and their ability to adapt in captivity and consume balanced feeds (Álvarez-Lajonchère and Tsuzuki 2008, Labastida-Che et al. 2013). Today, in Mexico, natural populations of snook species are overexploited (Arreguín-Sánchez and Arcos-Huitrón 2011), so biotechnological developments have begun to be made at the marine fish production pilot plant at the Research Center for Food and Development (CIAD, for its acronym in Spanish), Mazatlán (Sinaloa, Mexico), for the production of high-quality C. viridis juveniles to promote the farming of this very important species in Mexico and to develop repopulation programs (Ibarra-Castro et al. 2017).

In the marine fish farming industry, the highest operational cost is feed, so proper design of the feeding strategy is critical for a successful mariculture enterprise (Baloi et al. 2016, D'Abramo 2019). Feeding frequency affects growth, food intake, feed conversion rate and efficiency, body chemical composition, fish survival, and the quality of farming water (Biswas et al. 2010, Shipton and Hasan 2013, CostaBomfim et al. 2014, Rahman and Lee 2017). Juvenile marine fish need high feeding frequencies on a daily basis to attain to good performance in the culture (Schnaittacher et al. 2005, da Cunha et al. 2013, Luo et al. 2015); however, overfeeding reduces feed efficiency, increases lipid accumulation, mainly in liver and viscera, deteriorates the quality of farming water, and increases production costs (Lee and Pham 2010, Mizanur and Bai 2014, Lee et al. 2016, Guo et al. 2018). On the other hand, low feeding frequency does not provide the required nutrients for normal growth and survival of fish and, therefore, promotes size dispersion and cannibalism; furthermore, it can cause oxidative damage and immunosuppression (Tucker et al. 2006, Oh and Venmathi-Maran 2015, Tian et al. 2015). Several authors have reported optimal feeding frequencies that improve food intake, digestion, nutrient absorption, growth, and survival in juveniles marine fishes such as Limanda ferruginea (Dwyer et al. 2002), Pseudosiaena crocea (Xie et al. 2011), Trachinotus ovatus (Wu et al. 2015), Trachinotus blochii (Hamed et al. 2016), Megalobrama amblycephala (Xu et al. 2016), Sebastes inermis (Oh et al. 2018). Likewise, it has been reported that optimal feeding frequency depends on

\section{INTRODUCCIÓN}

El robalo blanco del pacífico (Centropomus viridis) se distribuye desde Baja California Sur y el golfo de California hasta el sur de Ecuador y las islas Galápagos (Fischer et al. 1995). Las especies de la familia Centropomidae habitan en aguas costeras someras, estuarios, ríos y lagunas salobres, y realizan migraciones a aguas dulces. Es muy común encontrar juveniles y preadultos en áreas de manglar, donde muestran gran tolerancia a las fluctuaciones de salinidad debido a su capacidad de osmorregulación (Álvarez-Lajonchère et al. 2013). Los robalos se consideran especies con alto potencial de cultivo en México porque tienen alto valor económico y gran demanda en el mercado nacional debido al sabor, color y textura de su carne; presentan altas tasas de crecimiento; y pueden adaptarse al cautiverio y consumir alimento balanceado (Álvarez-Lajonchère y Tsuzuki 2008, Labastida-Che et al. 2013). Actualmente, en México, las poblaciones naturales de las especies de robalo se encuentran sobreexplotadas (Arreguín-Sánchez y Arcos-Huitrón 2011), por lo que en la planta piloto para la producción de peces marinos del Centro de Investigación en Alimentación y Desarrollo (CIAD), unidad Mazatlán (Sinaloa, México), se ha iniciado el desarrollo de la biotecnología para la producción de juveniles de calidad de C. viridis para impulsar el cultivo de esta especie tan importante en México y desarrollar programas de repoblación (Ibarra-Castro et al. 2017).

En la industria del cultivo de peces marinos, el costo operacional más alto es la alimentación, por lo que el diseño apropiado de la estrategia alimenticia es fundamental para el éxito de la maricultura (Baloi et al. 2016, D'Abramo 2019). La frecuencia alimenticia influye en el crecimiento, el consumo de alimento, la tasa de conversión y eficiencia alimenticia, la composición química del cuerpo y la supervivencia de los peces, así como en la calidad del agua de cultivo (Biswas et al. 2010, Shipton y Hasan 2013, CostaBomfim et al. 2014, Rahman y Lee 2017). Se ha demostrado que los juveniles de peces marinos necesitan, diariamente, altas frecuencias de alimentación para obtener un buen rendimiento en el cultivo (Schnaittacher et al. 2005, da Cunha et al. 2013, Luo et al. 2015); sin embargo, la sobrealimentación reduce la eficiencia alimenticia, incrementa la acumulación de lípidos, principalmente en hígado y vísceras, deteriora la calidad del agua de cultivo e incrementa los costos de producción (Lee y Pham 2010, Mizanur y Bai 2014, Lee et al. 2016, Guo et al. 2018). Por otro lado, una baja frecuencia de alimentación no proporciona los nutrientes necesarios para el crecimiento normal y la supervivencia de los peces y, por lo tanto, promueve un aumento en la dispersión de tallas y el canibalismo; además, puede provocar daño oxidativo e inmunosupresión (Tucker et al. 2006, Oh y Venmathi-Maran 2015, Tian et al. 2015). Varios autores han reportado la frecuencia de alimentación óptima para mejorar el consumo de alimento, la digestión, la absorción de nutrientes, el crecimiento y la supervivencia en juveniles 
the species, age, size, environmental factors, feed quality, and cultivation system (Hamed et al. 2016, Xu et al. 2016, Oh et al. 2018). There are currently no published data on the frequency and optimal feeding time for C. viridis; therefore, the objective of the present study was to determine the appropriate feeding frequency and time for optimal performance of $C$. viridis juveniles and, in this way, contribute to the development of farming biotechnology for the species.

\section{MATERIALS AND METHODS}

\section{Fish and experimental system}

Centropomus viridis juveniles were obtained from the marine fish production pilot plant at CIAD in Mazatlán. The experiment was carried out in the CIAD area for bioassays, in 24 circular fiberglass tanks with black walls and white bottoms, each with 600-L capacity. The tanks had a central drain made with a 50-mm diameter PVC pipe, which was covered with a 5-mm mesh size net to prevent fish from escaping and to facilitate cleaning of the tanks. Each tank was provided with aeration and a flow-through system (approximately $6 \mathrm{~L} \cdot \mathrm{min}^{-1}$ ), with individual flow control valves to regulate flux. Seawater was pumped from Brujas Beach, Mazatlán, and passed through sand filters and cartridge filters with relative retention rating of $16 \mu \mathrm{m}$.

\section{Experimental design}

A completely randomized one-factor (feeding frequency) experimental design with 3 replicates was used. This design included 8 treatments, which is the number of treatments obtained after combining the number of feedings per day and feeding times (morning, noon, and evening). The assessed treatments were $1 \mathrm{M}, 1 \mathrm{~T}, 2 \mathrm{MD}, 2 \mathrm{DT}, 2 \mathrm{MT}, 3 \mathrm{MDT}, 3 \mathrm{DT}$, and 5MDT (Table 1). Each tank contained 20 juveniles weighing $0.36 \pm 0.01 \mathrm{~g}$, on average, which were fed a commercial feed for juvenile marine fish ad libitum for 6 weeks (Skretting, $0.8-1.5 \mathrm{~mm}$ ), according to the dietary regimen (Table 1) that was randomly selected.

\section{Experimental conditions and development}

Daily food intake by juveniles and the temperature, salinity, and dissolved oxygen of water in each tank were recorded. The bottom of the tanks was siphoned daily to remove feces and food remains. During the 6 weeks of the bioassay, water temperature was kept at $29 \pm 0.05^{\circ} \mathrm{C}$, salinity at $34 \pm 1.0$, and dissolved oxygen at $5.5 \pm 0.3 \mathrm{mg} \cdot \mathrm{L}^{-1}$.

To assess growth, at the end of the experiment, all juveniles from each replica were individually sedated with clove essence $\left(0.2 \mathrm{~mL} \cdot \mathrm{L}^{-1}\right)$, and after removing excess moisture with blotting paper, each individual was weighed on a digital scale with $\pm 0.05 \mathrm{~g}$ precision and measured for total length de peces marinos como en Limanda ferruginea (Dwyer et al. 2002), Pseudosiaena crocea (Xie et al. 2011), Trachinotus ovatus (Wu et al. 2015), Trachinotus blochii (Hamed et al. 2016), Megalobrama amblycephala (Xu et al. 2016), Sebastes inermis (Oh et al. 2018), entre otros. Así mismo, se ha reportado que la frecuencia óptima de alimentación depende de la especie, la edad, la talla, los factores ambientales, la calidad del alimento y el sistema de cultivo (Hamed et al. 2016, Xu et al. 2016, Oh et al. 2018). Hasta la fecha, no existen datos publicados sobre la frecuencia y el tiempo óptimo de alimentación para C. viridis; por lo tanto, el presente trabajo tuvo como objetivo determinar la frecuencia y el tiempo de alimentación adecuados para el rendimiento de juveniles de $C$. viridis para, así, contribuir al desarrollo de la biotecnología de cultivo de la especie.

\section{MATERIALES Y MÉTODOS}

\section{Peces y sistema experimental}

Los juveniles de C. viridis se obtuvieron de la planta piloto para la producción de peces marinos del CIAD, Unidad Mazatlán. El experimento se realizó en el área de bioensayos del CIAD en 24 tanques circulares de fibra de vidrio con paredes negras y fondo blanco, con capacidad de $600 \mathrm{~L}$ cada uno. Los tanques estuvieron provistos de un drenaje central de tubo de PVC de $50 \mathrm{~mm}$ de diámetro, cubierto con malla con una luz de $5 \mathrm{~mm}$ para evitar la salida de los peces y a la vez permitir la limpieza de los tanques. Cada tanque contó con aireación y flujo de agua continuo $\left(6 \mathrm{~L} \cdot \mathrm{min}^{-1}\right.$, aproximadamente), a través de llaves individuales de control de caudal para regular el flujo. El agua de mar se bombeó desde playa Brujas, Mazatlán, y fue pasada a través de filtros de arena y de cartuchos de $16 \mu \mathrm{m}$ de retención relativa.

\section{Diseño experimental}

Se empleó un diseño experimental completamente aleatorizado de un factor (frecuencia de alimentación) con 3 réplicas. Este diseño consideró 8 tratamientos, como resultado de la combinación del número de alimentaciones por día y la hora del día que se suministró (mañana, mediodía $\mathrm{y}$ tarde). Los tratamientos evaluados fueron $1 \mathrm{M}, 1 \mathrm{~T}, 2 \mathrm{MD}$, 2DT, 2MT, 3MDT, 3DT y $5 \mathrm{MDT}$ (Tabla 1). En cada tanque se colocaron 20 juveniles con peso promedio de $0.36 \pm 0.01 \mathrm{~g}$, los cuales se alimentaron a ad libitum durante 6 semanas con un alimento comercial para juveniles de peces marinos (Skretting, de 0.8 a $1.5 \mathrm{~mm}$ ), de acuerdo con el régimen de alimentación (Tabla 1) seleccionado al azar.

\section{Condiciones y desarrollo del experimento}

Se registró el consumo diario de alimento de los juveniles de cada tanque, así como la temperatura, la salinidad y el oxígeno disuelto en el agua. El fondo de los tanques se 
(TL) with a conventional vernier. Gained weight (GW), growth rate (GR), specific growth rate (SGR), and coefficient of variation $(\mathrm{CV})$ were calculated using the following formulas:

$$
\begin{aligned}
& \text { GW }(\mathrm{g})=\text { Average final weight }(\mathrm{FW}) \\
& \text { - Average initial weight (IW) ' } \\
& \mathrm{GR}(\%)=\frac{\mathrm{GW}}{\mathrm{IW}} \times 100, \\
& \text { SGR }\left(\% \mathrm{~d}^{-1}\right)=100 \times \frac{(\ln \text { weight at that time }-\ln \mathrm{IW})}{\text { Time }}, \\
& \mathrm{CV} \text { weight }(\%)=\frac{\text { Standard deviation of } \mathrm{FW}}{\mathrm{FW}} \times 100
\end{aligned}
$$

Feeding efficiency was determined using the food conversion ratio (FCR), feeding efficiency rate (FER), hepatosomatic index $(\mathrm{HI})$, and peritoneal fat index (PFI):

$$
\begin{gathered}
\text { FCR }=\frac{\text { Food intake }(\mathrm{FI})}{\mathrm{GW}}, \\
\text { FER }(\%)=\frac{\mathrm{GW}}{\mathrm{FI}} \times 100, \\
\mathrm{HI}=\frac{\text { Weight of liver }}{\mathrm{FW}} \times 100, \\
\mathrm{PFI}=\frac{\text { Wet weight of fat }}{\mathrm{FW}} \times 100 .
\end{gathered}
$$

The survival percentage was calculated (S):

$$
\mathrm{S}(\%)=\frac{\text { Final } N \text { fish }}{\text { Initial } N \text { fish }} \times 100
$$

\section{Statistical analysis of data}

Percentage values were arcsine transformed to homogenize variances. All results were tested for normality (Bartlett's test) and homoscedasticity (Levene's test). Normal and homoscedastic data (FW, GW, GR, SGR, CV, FI, FCR, and FER) were analyzed using a one-way analysis of variance (ANOVA, $P<0.05$ ), and significant differences between treatments were determined by Tukey's multiple rank comparison tests $(\alpha=0.05)$; data not showing a normal distribution (HI and PFI) were analyzed using a Kruskal-Wallis test $(P<0.05)$ and significant differences were determined using Levene's test based on the median (Zar 1996). All statistical analyses were done using the Statgraphics Centurion XVI program v.16.204. sifoneó diariamente para eliminar heces y restos de alimento. Durante las 6 semanas del bioensayo, la temperatura del agua se mantuvo a $29 \pm 0.05{ }^{\circ} \mathrm{C}$, la salinidad a $34 \pm 1.0 \mathrm{y}$ el oxígeno disuelto a $5.5 \pm 0.3 \mathrm{mg} \cdot \mathrm{L}^{-1}$.

Para evaluar el crecimiento, al final del experimento, todos los juveniles de cada réplica se anestesiaron individualmente con esencia de clavo $\left(0.2 \mathrm{~mL} \cdot \mathrm{L}^{-1}\right)$, y después de eliminar la humedad excesiva con papel secante, cada uno fue pesado en una balanza digital con precisión de $\pm 0.05 \mathrm{~g}$ y medido a longitud total (LT) con un vernier convencional. Se calculó el peso ganado (PG), la tasa de crecimiento (TC), la tasa específica de crecimiento (TEC) y el coeficiente de variación $(\mathrm{CV})$ mediante las siguientes fórmulas:

$$
\begin{gathered}
\text { PG }(\mathrm{g})= \\
\text { Promedio del peso final }(\mathrm{PF}) \\
- \text { Promedio del peso inicial }(\mathrm{PI}) \\
\mathrm{TC}(\%)=\frac{\mathrm{PG}}{\mathrm{PI}} \times 100 \\
\mathrm{TEC}\left(\% \mathrm{~d}^{-1}\right)=100 \times \frac{(\ln \text { peso en ese tiempo }-\ln \mathrm{PI})}{\text { Tiempo }} \\
\mathrm{CV} \text { peso }(\%)=\frac{\text { Desviación estándar del PF }}{\mathrm{PF}} \times 100 .
\end{gathered}
$$

La eficiencia alimenticia se determinó mediante la tasa de conversión alimenticia (TCA), la tasa de eficiencia alimenticia (TEA), el índice hepatosomático (IH) y el índice de grasa peritoneal (IGP):

$$
\begin{gathered}
\mathrm{TCA}=\frac{\text { Alimento consumido }(\mathrm{AC})}{\mathrm{PG}}, \\
\mathrm{TEA}(\%)=\frac{\mathrm{PG}}{\mathrm{AC}} \times 100 \\
\mathrm{IH}=\frac{\text { Peso del hígado }}{\mathrm{PF}} \times 100
\end{gathered}
$$

$$
\mathrm{IGP}=\frac{\text { Peso húmedo de grasa }}{\mathrm{PF}} \times 100 .
$$

Se calculó el porcentaje de supervivencia (S):

$$
\mathrm{S}(\%)=\frac{N \text { final de peces }}{N \text { inicial de peces }} \times 100
$$

\section{Análisis estadístico de datos}

Los resultados en porcentaje fueron transformados en arcoseno para homogeneizar las varianzas. Se verificó la normalidad (prueba de Bartlett) y la homocedasticidad 
Abdo-de la Parra et al.: Effect of feeding frequency on Centropomus viridis juveniles

Table 1. Experimental design (modified from Van der Meer et al. 1997). Tested feeding frequencies and times for Centropomus viridis juveniles.

Tabla 1. Diseño experimental (modificado de van der Meer et al. 1997). Frecuencias y horarios de alimentación evaluados para juveniles de Centropomus viridis.

\begin{tabular}{|c|c|c|c|c|c|}
\hline \multirow{2}{*}{$\begin{array}{l}\text { Feeding } \\
\text { frequency } \\
\text { (Treatment code) }\end{array}$} & \multicolumn{5}{|c|}{ Feeding time (h) } \\
\hline & $7: 00$ & $10: 00$ & $13: 00$ & $16: 00$ & $19: 00$ \\
\hline $\mathrm{M}$ & $\mathrm{X}$ & & & & \\
\hline $1 \mathrm{~T}$ & & & & & $\mathrm{X}$ \\
\hline $2 \mathrm{MD}$ & $X$ & & $\mathrm{X}$ & & \\
\hline 2DT & & & $\mathrm{X}$ & & $\mathrm{X}$ \\
\hline $2 \mathrm{MT}$ & $X$ & & & & $\mathrm{X}$ \\
\hline $3 \mathrm{MDT}$ & $\mathrm{X}$ & & $\mathrm{X}$ & & $\mathrm{X}$ \\
\hline 3DT & & $\mathrm{X}$ & $\mathrm{X}$ & $\mathrm{X}$ & \\
\hline $5 \mathrm{MDT}$ & $\mathrm{X}$ & X & $\mathrm{X}$ & $\mathrm{X}$ & $\mathrm{X}$ \\
\hline
\end{tabular}

$\mathrm{M}=$ morning, $\mathrm{T}=$ evening, $\mathrm{D}=$ noon.

\section{RESULTS}

FW and GW were significantly higher (ANOVA, $P=$ 0.0000 ) in juveniles fed 5 times a day (5MDT). GR and SGR values for juveniles fed 3 times a day, regardless of feeding time (3MDT and 3DT), did not show significant differences (ANOVA, $P>0.05$ ) with respect to the results obtained for organisms in the 5MDT treatment. Fish fed only once or twice a day, either in the morning (1M, 2MD) or in the evening (1T, 2DT), showed significantly less growth (ANOVA, $P=0.0000)$ than the rest of the juveniles in the other treatments. Growth in juveniles fed 2 times a day in the morning and the evening (2MT) was not significantly different (ANOVA, $P>0.05$ ) from growth in juveniles fed 3 times a day (3MDT and 3DT). The CV showed no significant differences (ANOVA, $P=0.7548$ ) between treatments (Table 2).

Regarding feeding efficiency, juveniles in the $1 \mathrm{M}$ and $1 \mathrm{~T}$ treatments showed significantly higher FCR (ANOVA, $P=$ 0.0009 ) and significantly lower FER (ANOVA, $P=0.0009$ ) in comparison with juveniles in the rest of the treatments. HI showed no significant differences (Kruskal-Wallis, $P=$ 0.3928 ) between treatments. PFI was significantly different (Kruskal-Wallis, $P=0.00029$ ) only between juveniles in the $5 \mathrm{MDT}$ treatment and juveniles in the $1 \mathrm{M}$ and $1 \mathrm{~T}$ treatments. Regarding survival, no significant differences $(P<0.05)$ were observed between treatments (Table 3).

\section{Discussion}

Several studies have shown that growth and feeding efficiency increase when feeding frequency is increased up to a certain number of times (Ribeiro et al. 2015, Baloi et (prueba de Levene) de todos los resultados. Los datos normales y homocedásticos (PF, PG, TC, TEC, CV, AC, TCA y TEA) se analizaron mediante un análisis de varianza de una vía (ANDEVA, $P<0.05$ ), y las diferencias significativas entre los tratamientos se determinaron por pruebas de comparación múltiple de rangos de Tukey $(\alpha=0.05)$; los datos que no presentaron distribución normal (IH e IGP) se analizaron mediante una prueba de Kruskal-Wallis $(P<0.05)$ y las diferencias significativas se determinaron mediante contraste de medianas de Levene (Zar 1996). Todos los análisis estadísticos se realizaron mediante el programa Statgraphics Centurion XVI v.16.204.

\section{Resultados}

El PF y el PG fueron significativamente más altos (ANDEVA, $P=0.0000$ ) en los juveniles alimentados 5 veces al día (5MDT). La TC y la TEC de los juveniles alimentados 3 veces al día, independientemente de las horas de alimentación (3MDT y 3DT), no presentaron diferencias significativas (ANDEVA, $P>0.05$ ) con respecto a los resultados obtenidos para los organismos del tratamiento 5MDT. Los peces que se alimentaron una sola vez o 2 veces al día, ya sea en la mañana (1M, 2MD) o en la tarde (1T, 2DT), presentaron un crecimiento significativamente más bajo (ANDEVA, $P=$ 0.0000 ) que el resto de los juveniles en los otros tratamientos. El crecimiento de los juveniles alimentados 2 veces al día por la mañana y por la tarde (2MT) no fue significativamente diferente (ANDEVA, $P>0.05$ ) del crecimiento de los juveniles alimentados 3 veces al día (3MDT y 3DT). El CV no presentó diferencias significativas (ANDEVA, $P=0.7548$ ) entre los tratamientos (Tabla 2). 
al. 2016, Guo et al. 2018, Oh et al. 2019). The present study showed that growth (FCR and FER) in C. viridis juveniles increased significantly when they were fed 3 times a day and that growth was not significantly different when they were fed 5 times a day. These results are similar to those reported by Mendes-de-Oliveira et al. (2019), who tested 2 to 6 feeding frequencies per day in Centropomus undecimalis and concluded that juveniles should be fed 4 times a day for adequate growth. Moreover, Tian et al. (2015) assessed the effect of 1 to 6 feeding frequencies per day on the growth of Megalobrama amblycephala juveniles and determined that optimal feeding frequency was 3 times a day. On the contrary, if feeding frequency is reduced to 1 or 2 times a day, growth will decrease because fish cannot obtain the necessary nutrients and meet the energy requirements for their performance and somatic development; likewise, FER will decrease and FCR will increase (Salama 2008, Biswas et al. 2010, Lee and Pham 2010, Hamed et al. 2016). This was observed in this study when C. viridis juveniles fed 1 or 2 times a day showed lower GW, GR, and SGR compared with fish fed 3 and 5 times a day. FCR was significantly higher and FER was significantly lower in juveniles fed once a day. Increasing feeding frequency, on the other hand, has been reported to produce uniform fish sizes, probably because small fish have a better chance of consuming food and competition between fish in the same tank decreases (Mihelakakis et al. 2002, Tucker et al. 2006, Biswas et al. 2010, Ribeiro et al. 2015). However, in the present study, no significant differences were observed in $\mathrm{CV}$ values for weight between the different treatments, which has also been observed for $C$. undecimalis (Mendes-de-Oliveria et al. 2019) and other marine fish species such as L. ferruginea (Dwyer et al. 2002),
Con respecto a la eficiencia alimenticia, se observó que en los juveniles de los tratamientos $1 \mathrm{M}$ y $1 \mathrm{~T}$, la TCA fue significativamente mayor (ANDEVA, $P=0.0009$ ) y la TEA fue significativamente menor (ANDEVA, $P=0.0009$ ), en relación con los juveniles del resto de los tratamientos. No se encontraron diferencias significativas (Kruskal-Wallis, $P=0.3928)$ en el IH entre tratamientos. El IGP solo presentó diferencias significativas (Kruskal-Wallis, $P=0.00029$ ) entre los juveniles del tratamiento 5MDT y los juveniles de los tratamientos $1 \mathrm{M}$ y $1 \mathrm{~T}$. En cuanto a la supervivencia, no se observaron diferencias significativas $(P<0.05)$ entre los tratamientos evaluados (Tabla 3 ).

\section{Discusión}

Varios estudios han demostrado que, al incrementar la frecuencia de alimentación hasta un cierto número de veces, se incrementa el crecimiento y la eficiencia alimenticia (Ribeiro et al. 2015, Baloi et al. 2016, Guo et al. 2018, Oh et al. 2019). En el presente estudio, se observó que el crecimiento (TCA y TEA) de los juveniles de C. viridis incrementó significativamente al alimentarlos 3 veces al día y se demostró que no hay diferencias significativas en el crecimiento si se alimentan 5 veces al día. Estos resultados son similares a los reportados por Mendes-de-Oliveira et al. (2019), quienes evaluaron de 2 a 6 frecuencias de alimentación por día en Centropomus undecimalis y concluyeron que los juveniles deben ser alimentados 4 veces al día para obtener un adecuado crecimiento. Por su parte, Tian et al. (2015) evaluaron de 1 a 6 frecuencias de alimentación al día en el crecimiento de juveniles de Megalobrama amblycephala y determinaron que la frecuencia óptima de alimentación fue de 3 veces por día. En el caso contrario, si se reduce la frecuencia de alimentación

Table 2. Growth performance of juvenile Centropomus viridis during the experiment. IW, initial average weight; FW, final average weight; $\mathrm{GW}$, gained weight; GR, growth rate; SGR, specific growth rate; CV, coefficient of variation.

Tabla 2. Crecimiento de los juveniles de Centropomus viridis durante el experimento. IW, promedio del peso al inicio; FW, promedio del peso al final; GW, peso ganado; GR, tasa de crecimiento; SGR, tasa específica de crecimiento; CV, coeficiente de variación.

\begin{tabular}{lcccccc}
\hline Treatment & IW $(\mathrm{g})$ & FW $(\mathrm{g})$ & GW $(\mathrm{g})$ & GR $(\%)$ & SGR $\left(\% \mathrm{~d}^{-1}\right)$ & CV $(\%)$ \\
\hline $1 \mathrm{M}$ & $0.35 \pm 0.02$ & $2.79 \pm 0.20^{\mathrm{a}}$ & $2.43 \pm 0.20^{\mathrm{a}}$ & $685.98 \pm 13.60^{\mathrm{a}}$ & $4.59 \pm 0.10^{\mathrm{a}}$ & $31.00 \pm 6.00$ \\
$1 \mathrm{~T}$ & $0.34 \pm 0.01$ & $2.70 \pm 0.10^{\mathrm{a}}$ & $2.35 \pm 0.10^{\mathrm{a}}$ & $676.50 \pm 66.80^{\mathrm{a}}$ & $4.56 \pm 0.02^{\mathrm{a}}$ & $33.33 \pm 4.60$ \\
2MD & $0.37 \pm 0.01$ & $4.47 \pm 0.30^{\mathrm{b}}$ & $4.09 \pm 0.30^{\mathrm{b}}$ & $1064.04 \pm 93.80^{\mathrm{b}}$ & $5.50 \pm 0.20^{\mathrm{b}}$ & $34.66 \pm 2.00$ \\
2DT & $0.35 \pm 0.01$ & $4.49 \pm 0.10^{\mathrm{b}}$ & $4.13 \pm 0.10^{\mathrm{b}}$ & $1173.07 \pm 103.40^{\mathrm{b}}$ & $5.66 \pm 0.10^{\mathrm{b}}$ & $30.00 \pm 6.00$ \\
2MT & $0.36 \pm 0.02$ & $4.98 \pm 0.40^{\mathrm{bc}}$ & $4.61 \pm 0.50^{\mathrm{bc}}$ & $1247.93 \pm 185.60^{\mathrm{bc}}$ & $5.78 \pm 0.30^{\mathrm{bc}}$ & $27.33 \pm 5.10$ \\
3MDT & $0.36 \pm 0.01$ & $5.97 \pm 0.70^{\mathrm{c}}$ & $5.60 \pm 0.60^{\mathrm{c}}$ & $1519.45 \pm 115.90^{\text {cd }}$ & $6.23 \pm 0.10^{\text {cd }}$ & $32.66 \pm 3.00$ \\
3DT & $0.34 \pm 0.02$ & $5.65 \pm 0.60^{\mathrm{bc}}$ & $5.29 \pm 0.60^{\mathrm{bc}}$ & $1506.00 \pm 173.70^{\text {cd }}$ & $6.19 \pm 0.20^{\text {cd }}$ & $34.33 \pm 8.10$ \\
5MDT & $0.38 \pm 0.01$ & $7.32 \pm 0.40^{\mathrm{d}}$ & $7.00 \pm 0.30^{\mathrm{d}}$ & $1831.29 \pm 75.47^{\mathrm{d}}$ & $6.57 \pm 0.10^{\mathrm{d}}$ & $32.33 \pm 5.50$ \\
\hline
\end{tabular}

Values (mean $\pm \mathrm{SD}, n=3$ ) with different letters in the same column are significantly different according to Tukey's multiple range test $(\alpha=0.05)$. 
Abdo-de la Parra et al.: Effect of feeding frequency on Centropomus viridis juveniles

Table 3. Food utilization, somatic indexes, and survival of juvenile white snook Centropomus viridis during the experiment. FI, food intake; FCR, food conversion ratio; FER, feeding efficiency rate; HI, hepatosomatic index; PFI, peritoneal fat index; S, survival.

Tabla 3. Eficiencia alimenticia, índices morfológicos y supervivencia de juveniles de robalo blanco Centropomus viridis durante el experimento. FI, alimento consumido; FCR, tasa de conversión alimenticia; FER, tasa de eficiencia alimenticia; HI, índice hepatosomático; PFI, índice de grasa peritoneal; $\mathrm{S}$, supervivencia.

\begin{tabular}{|c|c|c|c|c|c|c|}
\hline Treatment & FI (g) & FCR & FER (\%) & HI (\%) & PFI (\%) & $\mathrm{S}(\%)$ \\
\hline $1 \mathrm{~T}$ & $3.14 \pm 0.06^{\mathrm{a}}$ & $1.33 \pm 0.06^{\mathrm{b}}$ & $74.90 \pm 3.30^{\mathrm{a}}$ & $1.45 \pm 0.80$ & $2.04 \pm 0.40^{\mathrm{a}}$ & 100 \\
\hline $2 \mathrm{MD}$ & $4.49 \pm 0.40^{\mathrm{bc}}$ & $1.09 \pm 0.07^{\mathrm{a}}$ & $91.23 \pm 6.90^{\mathrm{b}}$ & $1.55 \pm 0.10$ & $3.25 \pm 1.00^{\mathrm{ab}}$ & $96.66 \pm 2.80$ \\
\hline 2DT & $4.19 \pm 0.10^{\mathrm{abc}}$ & $1.01 \pm 0.05^{\mathrm{a}}$ & $97.24 \pm 2.40^{\mathrm{b}}$ & $2.52 \pm 1.90$ & $2.96 \pm 0.70^{\mathrm{ab}}$ & 100 \\
\hline 3MDT & $5.82 \pm 0.20^{\mathrm{d}}$ & $1.04 \pm 0.16^{\mathrm{a}}$ & $96.70 \pm 16.00^{\mathrm{b}}$ & $1.50 \pm 0.70$ & $2.99 \pm 1.90^{\mathrm{ab}}$ & 100 \\
\hline $3 \mathrm{DT}$ & $5.13 \pm 0.60^{\mathrm{cd}}$ & $0.96 \pm 0.07^{\mathrm{a}}$ & $103.6 \pm 8.10^{\mathrm{b}}$ & $1.56 \pm 0.60$ & $3.22 \pm 0.40^{\mathrm{ab}}$ & $96.66 \pm 2.80$ \\
\hline $5 \mathrm{MDT}$ & $6.96 \pm 0.10^{\mathrm{e}}$ & $1.00 \pm 0.04^{\mathrm{a}}$ & $98.03 \pm 1.80^{b}$ & $1.38 \pm 0.40$ & $4.18 \pm 0.90^{\mathrm{b}}$ & 100 \\
\hline
\end{tabular}

Values (mean $\pm \mathrm{SD}, n=3$ ) with different letters in the same column are significantly different according to Tukey's multiple range test ( $\alpha=$ 0.05), except for IGP, which follows Levene's median contrast.

Pagrus auratus (Booth et al. 2008), and Platichthys flesus luscus (Küçük et al. 2014).

Regarding feeding time, the present study showed that there is no significant effect on juvenile $C$. viridis growth and feeding efficiency if feeding is done in the morning or the evening. This finding was also reported for Sigamus rivulatus (Barakat et al. 2011) and Colossoma macropomum (van der Meer et al. 1997). However, in some species, such as P. flesus luscus (Küçük et al. 2014) and Oplegnathus fasciatus (Oh and Venmathi-Maran 2015), reports show that food intake is higher in the first hour of the morning and in other species, such as Sardinella brasiliensis (Baloi et al. 2016) and Hapalogenys nigripinnis (Oh et al. 2019), intake is higher in the evening. In the present study, the intake of food supplied during each feeding was not evaluated, only total consumption per day, so it was not possible to determine the time of day with the highest food intake. In this study, HI in all treatments was not significantly affected by feeding frequency, and PFI was significantly higher in juveniles from the 5MDT treatment than in juveniles fed only once a day, regardless of feeding time (1M and $1 \mathrm{~T})$. Low PFI values suggest that during feed restriction, fish use stored fat to meet the energy requirements for growth (Baloi et al. 2016), which would explain why in the present study fish fed only once a day (1M and $1 \mathrm{~T})$ showed lower PFI values than fish fed 5 times a day (5MDT), since after having had insufficient food, they were possibly unable to store as much peritoneal fat as fish in the 5MDT treatment. Other studies on marine fish have also reported that PFI is affected by feeding frequency, the higher the feeding frequency, the higher the PFI values (Mizanur and Bai 2014, Baloi et al. 2016, Guo et al. 2018). a 1 o 2 veces al día, el crecimiento será menor, debido a que los peces no pueden obtener los requerimientos energéticos y los nutrientes necesarios para su mantenimiento y desarrollo somático; además, disminuiría la TEA y aumentaría la TCA (Salama 2008, Biswas et al. 2010, Lee y Pham 2010, Hamed et al. 2016). Lo mismo se observó en este estudio, ya que los juveniles de $C$. viridis alimentados 1 o 2 veces al día presentaron menor PG, TC y TCE en relación con los peces alimentados 3 y 5 veces al día. En cuanto a la TCA, esta fue significativamente mayor y la TEA fue significativamente menor en los juveniles alimentados 1 vez al día. Por otro lado, se ha reportado que al aumentar la frecuencia de alimentación, se producen peces de talla uniforme, probablemente debido a que los peces pequeños tienen más oportunidad de consumir alimento y disminuye la competencia entre los peces del mismo tanque (Mihelakakis et al. 2002, Tucker et al. 2006, Biswas et al. 2010, Ribeiro et al. 2015). Sin embargo, en el presente estudio no se observaron diferencias significativas en el CV del peso de los organismos entre los diferentes tratamientos, lo cual también ha sido observado en C. undecimalis (Mendes-de-Oliveria et al. 2019) y en otras especies de peces marinos como L. ferruginea (Dwyer et al. 2002), Pagrus auratus (Booth et al. 2008) y Platichthys flesus luscus (Küçük et al. 2014).

En cuanto a la hora de alimentación, el presente estudio demostró que no existe un efecto significativo en el crecimiento y la eficiencia alimenticia de los juveniles de $C$. viridis si la alimentación se lleva a cabo por la mañana o por la tarde. Este hallazgo también ha sido reportado para Sigamus rivulatus (Barakat et al. 2011) y Colossoma macropomum (van der Meer et al. 1997). Sin embargo, en algunas especies se ha reportado que el consumo de alimento es mayor en la 
From the results obtained in the present study, it can be concluded that, under the same experimental conditions, the optimal feeding frequency for pre-fattening C. viridis juveniles is 3 times a day within 6-hour intervals. These results will contribute to the development of farming biotechnology aiming to obtain adequate growth and feeding efficiency for this species, thus enabling operational cost optimization by using only the required feed, in addition to preventing organic waste accumulation due to unconsumed food.

\section{ACKNowledgments}

We thank Valerie Williams for her support in writing the English version of the abstract and correcting the manuscript, Juan Huerta for his technical assistance, and the CIAD marine fish production pilot plant staff for providing the snook juveniles. The authors state that there is no conflict of interest.

English translation by Claudia Michel-Villalobos.

\section{REFERENCES}

Álvarez-Lajonchère LS, Tsuzuki MY. 2008. A review of methods for Centropomus spp. (snooks) aquaculture and recommendations for the establishment of their culture in Latin America. Aquacult Res. 39(7):684-700. https://doi.org/10.1111/j.1365-2109.2008.01921.x

Álvarez-Lajonchère LS, Tsuzuki M, Ibarra-Castro L. 2013. Cultivo de robalos, familia Centropomidae. In: Castello-i-Orvay F (ed.), Piscicultura Marina en Latinoamérica: Bases científicas y técnicas para su desarrollo. Barcelona (Spain): Publicacions i Edicions de la Universitat de Barcelona. p. 231-245.

Arreguín-Sánchez F, Arcos-Huitrón E. 2011. La pesca en México: estado de la explotación y uso de los ecosistemas $=$ Fishing in Mexico: state of exploitation and use of ecosystems. Hidrobiológica. 21(3):431-462.

Baloi M, de Carvalho CVA, Sterzelecki FC, Passini G, Cerqueira VR. 2016. Effects of feeding frequency on growth, feed efficiency and body composition of juveniles Brazilian sardine, Sardinella brasiliensis (Steindacher 1879). Aquacult Res. 47(2):554-560. https://doi.org/10.1111/are.12514

Barakat A, Roumieh R, Meguid ANE, Ghanawi J, Saoud IP. 2011. Feed regimen affects growth, condition index, proximate analysis and myocyte ultrastructure of juvenile spinefoot rabbitfish Siganus rivulatus. Aquacut Nut. 17(3):e773-e780. https://doi.org/10.1111/j.1365-2095.2010.00847.x

Biswas G, Thirunavukkarasu AR, Sundaray JK, Kailasam M. 2010. Optimization of feeding frequency of Asian seabass (Lates calcarifer) fry reared in net cages under brackishwater environment. Aquaculture. 305:26-31.

https://doi.org/10.1016/j.aquaculture.2010.04.002

Booth MA, Tucker BJ, Allan GL, Fielder DS. 2008. Effect of feeding regime and fish size on weight gain, feed intake and gastric evacuation in juvenile Australian snapper Pagrus auratus. Aquaculture. 282(1-4):104-110. https://doi.org/10.1016/j.aquaculture.2008.06.027

Costa-Bomfim CN, Pessoa WVN, Oliveira RLM, Farias JL, Domingues EC, Hamilton S, Cavalli RO. 2014. The effect of feeding frequency on growth performance of juvenile cobia, Rachycentron canadum (Linnaeus, 1766). J Appl Ichthyol. 30:135-139.

https://oi.org/10.1111/jai.12339 primera hora de la mañana, como en P. flesus luscus (Küçük et al. 2014) y Oplegnathus fasciatus (Oh y Venmathi-Maran 2015), y en otras especies, como Sardinella brasiliensis (Baloi et al. 2016) y Hapalogenys nigripinnis (Oh et al. 2019), el consumo es mayor por la tarde. En el presente estudio no se evaluó el consumo de alimento suministrado en cada toma, solo el total consumido por día, por lo que no se pudo determinar la hora del día de mayor consumo de alimento. En este trabajo, el IH de todos los tratamientos no fue afectado significativamente por la frecuencia alimenticia, y el IGP fue significativamente mayor en los juveniles del tratamiento 5MDT que en los juveniles alimentados solo una vez al día, independientemente de la hora de alimentación (1M y 1T). Un IGP menor sugiere que durante la restricción de alimento, los peces utilizan la grasa acumulada para encontrar sus demandas energéticas necesarias para el crecimiento (Baloi et al. 2016), lo cual podría explicar por qué los peces alimentados solo $1 \mathrm{vez}$ al día (1M y 1T) en el presente estudio obtuvieron un IGP menor que los peces alimentados 5 veces al día (5MDT), ya que al no contar con suficiente alimento, posiblemente no pudieron acumular una mayor cantidad de grasa peritoneal como los peces en el tratamiento 5MDT. En otros estudios con peces marinos también se ha reportado que el IGP es afectado por la frecuencia de alimentación, a mayor frecuencia de alimentación, más altos los valores del IGP (Mizanur y Bai 2014, Baloi et al. 2016, Guo et al. 2018).

Con base en los resultados obtenidos en el presente estudio, se puede concluir que, bajo las mismas condiciones experimentales, la frecuencia de alimentación óptima para la pre-engorda de juveniles de $C$. viridis es de 3 veces al día con intervalos de $6 \mathrm{~h}$. Estos resultados contribuirán al desarrollo de la biotecnología de cultivo de esta especie, para obtener un adecuado crecimiento y eficiencia alimenticia, y se podrán optimizar los costos de operación al utilizar solo el alimento necesario, además de evitar la acumulación de desechos orgánicos por el alimento no consumido.

\section{Agradecimientos}

Agradecemos a Valerie Williams por su apoyo en la redacción del resumen en inglés y la corrección del manuscrito, a Juan Huerta por su asistencia técnica y al personal de la planta piloto para la producción de peces marinos del CIAD por proporcionar los juveniles de robalo. Los autores manifiestan que no existe algún conflicto de intereses.

D’Abramo L. 2019. Nutrition and Feeds. In: Lucas JS, Southgate PC, Tucker CS (eds.), Aquaculture: Farming Aquatic Animals and Plants. 3rd ed. Hoboken (NJ): John Wiley \& Sons Ltd. p. 157-182.

Da Cunha VL, Pereira-Shei MR, Okamoto MH, Vieira-Rodrigues R, Sampaio LA. 2013. Feeding rate and frequency on juvenile pompano growth. Pesq Agropec Bras. 48(8):950-954. http://doi.org/10.1590/S0100-204X2013000800020

Dwyer KS, Brown JA, Parrish C, Lall SP. 2002. Feeding frequency affects food consumption, feeding pattern and growth of 
juvenile yellowtail flounder(Limanda ferruginea). Aquaculture. 213(1-4):279-292. https://doi.org/10.1016/S0044-8486(02)00224-7

Fischer W, Krupp F, Schneider W, Sommer C, Carpenter KE, Niem VH. 1995. Guía FAO para la identificación de especies para los fines de la pesca. Pacífico centro-oriental. Vol. 2. Rome (Italy): FAO. Roma. 1,200 p.

Guo Z, Cui J, Li M, Liu H, Zhang M, Meng F, Shi G, Wang R, He $\mathrm{X}$, Zhao Y. 2018. Effect of feeding frequency on growth performance, antioxidant status, immune response and resistance to hypoxia stress challenge on juvenile dolly varden char Salvelinus malma. Aquaculture. 486:197-201. https://doi.org/10.1016/j.aquaculture.2017.12.031

Hamed SS, Jiddawi NS, Bwathondi POJ, Mmochi AJ. 2016. Effect of feeding frequency and feeding rate on growth performance of juvenile silver pompano, Trachinotus blochii. WIO J Mar Sci. 15(1):39-47.

Ibarra-Castro L, Navarro-Flores J, Sánchez-Téllez JL, MartínezBrown JM, Ochoa-Bojórquez LA, Rojo-Cebreros AH. 2017. Hatchery production of pacific white snook at CIAD-Unity Mazatlan, Mexico. World Aquaculture. 48(3):25-29.

Küçük E, Aydin I, Polat H, Eroldogan OT, Sahin T. 2014. Effect of feeding frequency on growth, feed efficiency and nutrient utilization of juvenile flounder (Platichthys flesus luscus). Aquacult Int. 22:723-732. https://doi.org/10.1007/s10499-013-9701-2

Labastida-Che A, Núñez-Orozco AL, Oviedo-Piamonte JA. 2013. Aspectos biológicos del robalo hocicudo Centropomus viridis, en el sistema lagunar Chantuto-Panzacola, Chiapas, México. Cienc Pesq. 21(2):21-28.

Lee SM, Pham MA. 2010. Effects of feeding frequency and feed type on the growth, feed utilization and body composition of juvenile olive flounder, Paralichthys olivaceus. Aquacult Res. 41(9):e166-e171. https://doi.org/10.1111/j.1365-2109.2010.02491.x

Lee S, Haller LY, Fangue NA, Fadel JG, Hung SSO. 2016. Effects of feeding rate on growth performance and nutrient partitioning of young-of-the-year white sturgeon (Acipenser transmontanus). Aquacult Nut. 22(2):400-409.

https://doi.org/10.1111/anu.12255

Luo L, Li T, Xing W, Xue M, Ma Z, Jiang N, Li W. 2015. Effects of feeding rates and feeding frequency on the growth performances of juvenile hybrid sturgeon, Acipenser schrenckii Brandt $+\times A$. baeri Brandt $\hat{\jmath}$. Aquaculture. 448:229-233. https://doi.org/10.1016/j.aquaculture.2015.06.005

Mendes-de-Oliveira RL, Gomes-dos-Santos LB, Gomes-da-SilvaNeto N, Alves-da-Silva SP, dos-santos-Silva F, Melatti E, Olivera-Cavalli RO. 2019. Feeding rate and feeding frequency affect growth performance of common snook (Centropomus undecimalis) juveniles reared in the laboratory. Rev Bras Zoo. 48:e20170292 https://doi.org/10.1590/rbz4820170292

Mihelakakis A, Tsolkas C, Yoshimatsu T. 2002. Optimization of feeding rate for hatchery-produced juvenile gilthead sea bream Sparus auruta. JWAS. 33(2):169-175. https://doi.org/10.1111/j.1749-7345.2002.tb00491.x

Mizanur RM, Bai SC. 2014. The optimum feeding frequency in growing Korean rockfish (Sebastes schlegeli) rearing at the temperature of $15^{\circ} \mathrm{C}$ and $19^{\circ} \mathrm{C}$. Asian Australas J Anim Sci. 27(9):1319-1327.

https://doi.org/10.5713/ajas.2014.14193

Oh SY, Venmathi-Maran BA. 2015. Feeding frequency influences growth, feed consumption and body composition of juvenile rock bream (Oplegnathus fasciatus). Aquacult Int. 23(1):175-184. https://doi.org/10.1007/s10499-014-9806-2
Oh SY, Venmathi-Maran BA, Park JW. 2018. Effect of feeding frequency on growth, food consumption, proximate composition, and blood chemistry of juvenile dark-banded rockfish, Sebastes inermis. JWAS. 49(6):994-1001. https://doi.org/10.1111/jwas.12512

Oh SY, Venmathi-Maran BA, Park JW. 2019. Optimum feeding frequency for juvenile short barbeled velvetchin Hapalogenys nigripinnis reared in floating sea cages. Fish Sci. 85(2):379-385. https://doi.org/10.1007/s12562-019-01288-1

Rahman MM, Lee SM. 2017. Effect of dietary lipid level and feeding frequency on the growth, feed utilization, and body composition of juvenile spotted seabass, Lateolabrax maculatus. JWAS. 48(4):634-642. https://doi.org/10.1111/jwas.12382

Ribeiro FF, Forsythe S, Qin JG 2015. Dynamics of intracohort cannibalism and size heterogeneity in juvenile barramundi (Lates calcarifer) at different stocking densities and feeding frequencies. Aquaculture. 444:55-61. http://doi.org/10.1016/j.aquaculture.2015.03.029

Salama AJ. 2008. Effects of different feeding frequency on the growth, survival and feed conversion ratio of the Asian sea bass Lates calcarifer juveniles reared under hypersaline seawater of the Red Sea. Aquacult Res. 39(6):561-567. https://doi.org/10.1111/j.1365-2109.2007.01890.x

Schnaittacher G, King-V W, Berlinsky DL. 2005. The effects of feeding frequency on growth of juvenile Atlantic halibut, Hippoglossus hippoglossus L. Aquacult Res. 36(4):370-377. https://doi.org/10.1111/j.1365-2109.2005.01218.x

Shipton TA, Hasan MR. 2013. An overview of the current status of feed management practices. In: Hasan MR, New MB (eds.), On farm feeding and feed management in aquaculture. FAO Fisheries and Aquaculture Technical Paper No. 583. Rome (Italy): FAO. p. 3-20.

Tian HY, Zhang DD, Li XF, Zhang CN, Qian Y, Liu WB. 2015. Optimum feeding frequency of juvenile blunt snout bream Megalobrama amblycephala. Aquaculture. 437:60-66. https://doi.org/10.1016/j.aquaculture.2014.11.032

Tucker BJ, Booth MA, Allan GL, Booth D, Fielder DS. 2006. Effects of photoperiod and feeding frequency on performance of newly weaned Australian snapper Pagrus auratus. Aquaculture. 258(1-4):514-520.

https://doi.org/10.1016/j.aquaculture.2006.03.033

Van der Meer MB, van Herwaarden H, Verdegem MCJ. 1997. Effect of number of meals and frequency of feeding on voluntary feed intake of Colossoma macropomum (Cuvier). Aquacult Res. 28(6):419-432. https://doi.org/10.1046/j.1365-2109.1997.00874.x

Xie F, Ai Q, Mai K, Xu W, Ma H. 2011. The optimal feeding frequency of large yellow croaker (Pseudosciaena crocea, Richardson) larvae. Aquaculture. 311(1-4):162-167. https://doi.org/10.1016/j.aquaculture.2010.12.005

Xu C, Li XF, Tian HY, Jiang GZ, Liu WB. 2016. Feeding rates affect growth, intestinal digestive and absorptive capabilities and endocrine functions of juvenile blunt snout bream Megalobrama amblycephala. Fish Physiol Biochem. 42(2):689-700. https://doi.org/10.1007/s10695-015-0169-Z

Wu Y, Han H, Qin J, Wang Y. 2015. Effect of feeding frequency on growth, feed utilization, body composition and waste output of juvenile golden pompano (Trachinotus ovatus) reared in net pens. Aquacult Res. 46(6):1436-1443.

https://doi.org/10.1111/are.12297

Received January 2020, accepted May 2020. 